\title{
ESC-Track: A computer workflow for 4-D segmentation, tracking, lineage tracing and dynamic context analysis of ESCs
}

\author{
Laura Fernández-de-Manúel, Covadonga Díaz-Díaz², Daniel Jiménez-Carretero, Miguel Torres², \\ and María C. Montoya ${ }^{1}$ \\ ${ }^{1}$ Cellomics Unit and ${ }^{2}$ Cardiovascular Developmental Program, Cell \& Developmental Biology Area, Centro \\ Nacional de Investigaciones Cardiovasculares CNIC, Madrid, Spain
}

BioTechniques 62:215-222 (May 2017) doi 10.2144/000114545

Keywords: ESC; image processing; segmentation; tracking; lineage

Supplementary material for this article is available at www.BioTechniques.com/article/114545.

Embryonic stem cells (ESCs) can be established as permanent cell lines, and their potential to differentiate into adult tissues has led to widespread use for studying the mechanisms and dynamics of stem cell differentiation and exploring strategies for tissue repair. Imaging live ESCs during development is now feasible due to advances in optical imaging and engineering of genetically encoded fluorescent reporters; however, a major limitation is the low spatio-temporal resolution of long-term 3-D imaging required for generational and neighboring reconstructions. Here, we present the ESC-Track (ESC-T) workflow, which includes an automated cell and nuclear segmentation and tracking tool for 4-D (3-D + time) confocal image data sets as well as a manual editing tool for visual inspection and error correction. ESC-T automatically identifies cell divisions and membrane contacts for lineage tree and neighborhood reconstruction and computes quantitative features from individual cell entities, enabling analysis of fluorescence signal dynamics and tracking of cell morphology and motion. We use ESC-T to examine Myc intensity fluctuations in the context of mouse ESC (mESC) lineage and neighborhood relationships. ESC$\mathrm{T}$ is a powerful tool for evaluation of the genealogical and microenvironmental cues that maintain ESC fitness.

Stem cells provide essential functions
during embryonic development and tissue
regeneration. Mouse embryonic stem cells
(mESCs) are derived from pluripotent cells
of the early mouse embryo and can be
maintained as stable cell lines with a high
self-renewal capacity. They provide a
versatile in vitro model for understanding
differentiation of human tissues, and their
study has led to major advances in cell
and developmental biology (1,2). A key
challenge in the field is to understand
the mechanisms involved in guiding stem
cell fate (3-5), which have broad appli-
cations in biomedicine, from elucidating
the causes of cancer to the use of stem
cells in regenerative medicine. Thus, the biological properties of ESCs are a matter of great scientific, commercial, and medical interest.

Optical imaging advances have led to the emergence of powerful live imaging tools with individual cell resolution in threedimensional (3-D) space and in time (3-D + time or 4-D) $(6,7)$. Moreover, a new generation of fluorescent proteins and dyes allows biochemical characterization of signaling pathways in intact living cells (8). Tagging by fluorescent proteins enables positional tracking of any given cell over time, which is easily achieved when the population of tagged cells is distributed among non-expressing cells by virtue of lineage or in experimental mosaics, but it becomes challenging when a fluorescent protein label is widely expressed (9). The ability to track and analyze live cells in time-lapse 4-D microscopy images is a matter of intense research $(10,11)$ since visual inspection and analysis are insufficient to extract meaningful insights, making automated tracking and quantitative analysis of cells an absolute requirement. This is such a challenging task that several competitions have been carried out in order to evaluate cell segmentation and tracking algorithms (12,13). Computational tools are essential for extracting quantitative measurements from stem cell populations growing in 3-D physiological conditions and to translate the measure-

\section{METHOD SUMMARY}

ESC-Track (ESC-T) is a computational tool for automated segmentation and tracking of single mouse embryonic stem cells (mESCs) from live-cell 4-D confocal image data sets. The ESC-T workflow enables the extraction of parameters related to fluorescence signal localization and dynamics, cellular morphology, and cell motion for individual cells in the context of lineage and neighborhood relationships. 
ments into biological knowledge, allowing the study of a range of cell behaviors, such as motility, cell division, death, phagocytosis, etc. Most of these methods have been applied to Drosophila (14-18) and zebrafish (19-21) embryogenesis, or plant morphogenesis (22) studies. Of special relevance to the field of stem cell biology is the ability to integrate the cell behavior analysis with information about lineage (parent-progeny) and contextual (neighborhood) cellular relationships $(9,11)$. In the last decade, several generic processing and tracking packages, such as Icy (23), Cell Profiler (24), tTt (25), qTfy (25), or the Fiji plugin TrackMate $(26,27)$ have been reported. Some complex methods have been developed for specific applications, such as MARS (22), ACME (21), EDGE-4-D (17), and RACE (18) for particle (28), nuclear $(16,29)$ or cellular $(17,18,20-22)$ segmentation, and STARRYNITE (29), U-TRACK (28), ALT (22), EDGE-4-D (17), and TGMM (16) for tracking.

Here, we present a computational workflow that allows the automated segmentation and tracking of individual mESCs from live-cell 4-D confocal image data sets based on the combination of membrane and non-homogeneous nuclear signals, allowing lineage and neighborhood reconstruction. The workflow enables the extraction of parameters for fluorescence signal localization and dynamics, cellular morphological characteristics, and motionrelated aspects of individual cells in the context of lineage and neighborhood relationships. ESC-T was used to study Myc dynamics in mESC cultures, and it proved to be a very valuable computational tool for stem cell research as it allowed the evaluation of genealogical and microenvironmental cues during mouse ESC culture in an unprecedented manner.

\section{Materials And Methods}

Image processing and analysis

Automatic cell and nuclei segmentation and cell tracking. The proposed pipeline (Figure 1) uses images obtained from ESCs expressing tdTomato and GFP-MYC signals as described in the Supplementary Material. The pre-processing step consists of median filtering combining both nuclear and membrane signals (mycGFP median minus tdTomato median) (Figure 1, Steps 1-4) and is followed by application of a 2-D watershed segmentation algorithm,

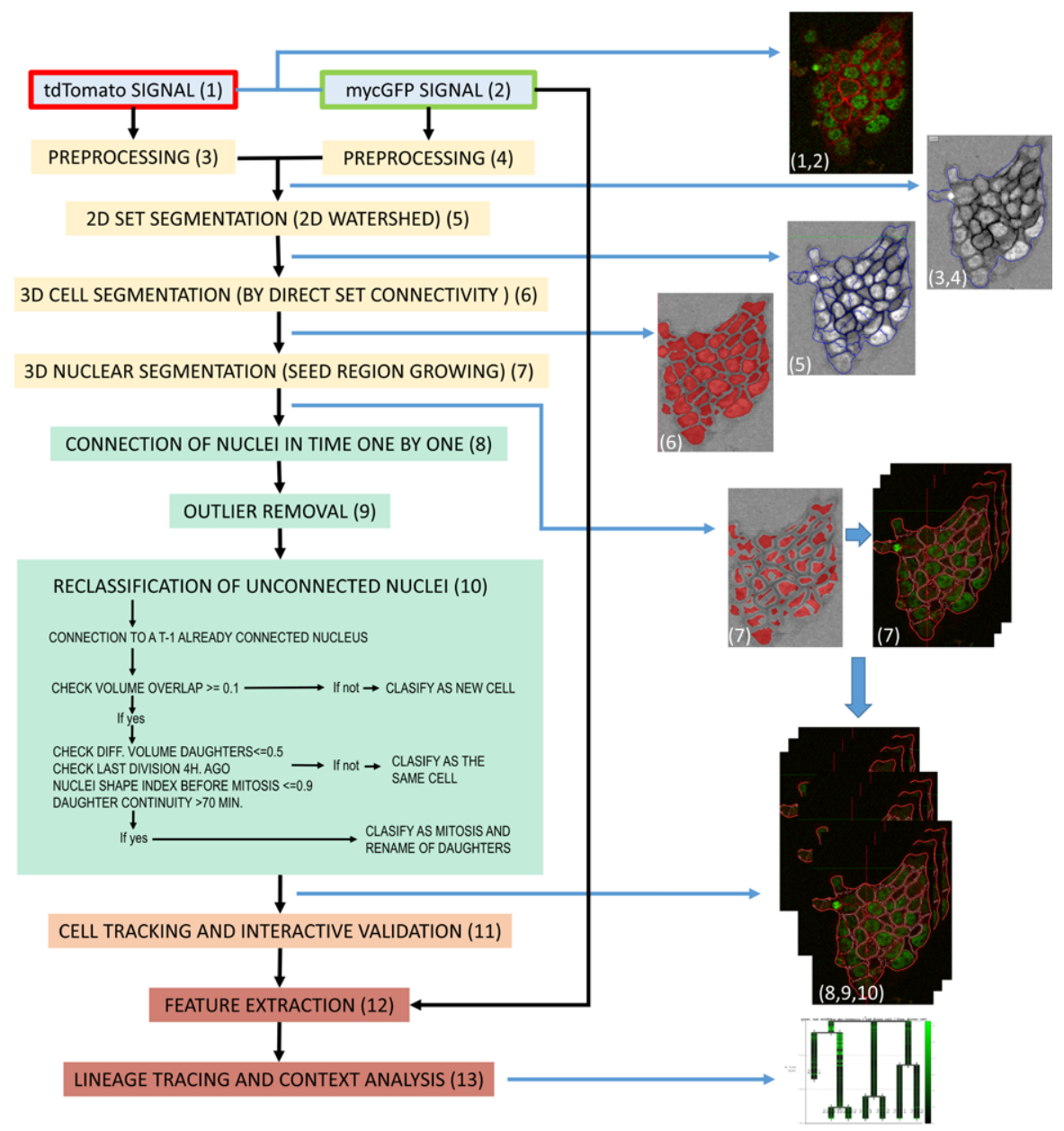

Figure 1. Segmentation and tracking computational pipeline.

rendering 2-D sets (cell portions) (Figure 1, Step 5). Spatiotemporal $(3-D+t)$ association rules based on the overlap of sets are applied to connect sets in 3-D space and time for automatic segmentation and tracking through the following pipeline:

(i) 3-D cells are defined by 3-D connection of 2-D sets based on direct connectivity (Figure 1, Step 6).

(ii) As non-homogenous nuclear staining prevents direct nuclear segmentation, a nuclei prediction image is generated by applying morphological erosion on the cellular segmentation mask and then combining it with a filtered version of the nuclear GFP-MYC channel (median filter with 2-D kernel of size 5). Seeds are automatically detected inside 3-D cells and develop following a 3-D region-growing algorithm for nuclei segmentation based on the nuclei prediction (Figure 1, Step 7).

(iii) Then, a hierarchical strategy uses an algorithmic loop on the overlap (ratio of the intersected area to the area of the current object) of 3-D nuclei to connect nuclei at time point $t$ with unconnected nuclei at time point $t-1$ or $t-2$ (Figure 1, Step 8). The threshold overlap value for connecting current and candidate nuclei is initially set at 0.7 . For the remaining unconnected nuclei, this threshold is reduced by 0.1 in a loop until it reaches 0.1.

(iv) Outliers with nuclei sizes (number of voxels) below a threshold, which is manually set by the user [typically 100 arbitrary units (a.u.)], are removed (Figure 1, Step 9).

(v) Nuclei remaining unconnected in time are re-analyzed for further classification (Figure 1, Step 10) as either pieces of preexisting cells, new cells arriving into the imaging volume, or newborn cells from cell division.

In order to identify non-highlighted cell divisions during automatic tracking, additional criteria are considered in spatiotemporal association rules, including biological knowledge about the minimum temporal distance between mitosis and nuclei shape before mitosis (30-32). 
A
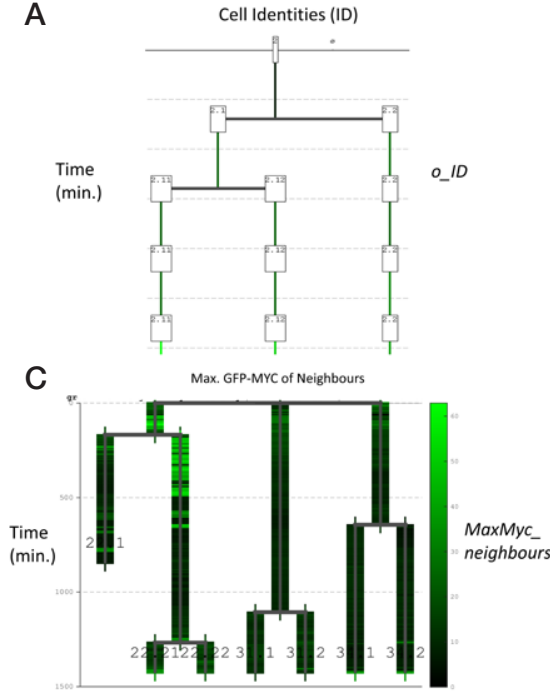

E

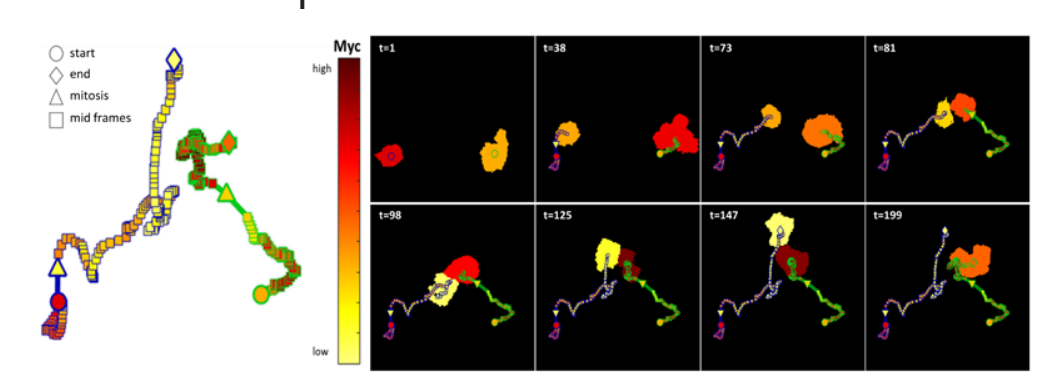

Figure 2. Lineage tree generated by the embryonic stem cell tracking workflow (ESC-T) and visualization of cell trajectories, contacts, and their relationship to Myc expression. (A) Assignment of cell identifiers (o_ID) to every tracked cell allows dividing cells to be connected with their progeny and represented as a lineage tree. The example shows the lineage tree of Progenitor 2 at five different time points, at which every cell entity is represented by a rectangle in which the cellular ID is indicated. (B-D) Lineage tree representation of extracted cellular features during tracking times (min). For every cell of the tree at each time point, a selected feature is represented as a color-coded rectangle to visually highlight changes in the feature along the chronological lineage traces. Panels show nuclear GFP-MYC intensities (B), nuclear maximum GFP-MYC intensity of neighbors (C), and nuclear volumes (D) for each cell in the lineage tree using the color code shown to the right of each chart. (E) Representation of motion tracks and Myc expression for two selected cells. The figure depicts trajectories and color-coded MYC-GFP normalized intensity values obtained at each time point using ESC-T. Cell position coordinates at each time point are represented with squares, which are colored according to the corrected mean_compensated_Mean_Myc score for the nucleus. Mitotic events and starting and final locations are represented by triangles, circles, and diamonds, respectively. Trajectories are highlighted in green and blue. (F) Video stills (from Supplementary Movie S1) obtained at the indicated time points, combining the Myc expression levels and trajectories as in (E).

If two nuclei at time point $t$ have been connected based on temporal overlap criteria to the same nucleus at $t-1$, they are considered to be candidate daughters resulting from cell division. In these cases, further criteria are applied:

(i) For each daughter candidate, the overlap with the mother cell (ratio of the intersected area) is $\geq 0.1$.

(ii) Volumes of candidate daughter cells relative to the mother cell are $\leq 0.5$.

(iii) Last division of mother cell is $>4 \mathrm{~h}$ earlier.

(iv) Nuclei shape index before cell division is $\leq 0.9$ (index ranging from 0 to 1 , with 0 indicating high smooth surface and roundness and 1 indicating (ow). (v) Separation of the cells maintained in time: Daughters appear as segmented cells $>70$ min after they are born.

If these cell division criteria are fulfilled, candidates are classified as newborn daughter cells, receiving a new identification number with information on their progenitor. If volume overlap $\geq 0.1$ for the 2 daughter candidates, but any of the other 3 conditions is not fulfilled, then both cells are considered as different sets of the same pre-existing cell and therefore merged and named with the same ID. If volume overlap $<0.1$ between a cell at $t 0$ and any other cell at $t-1$ or $t-2$, it is classified as a new cell arriving to culture. (Figure 1, Step 10). After classification, cell segmentation is updated with tracking feedback. IDs are structured as follows: the integer part represents the family, and the decimals identify daughters, with a decimal position being added at each generation (Figure 2A).

Manual edition and feature extraction. A visualization and editing interface was implemented to allow the user to correct errors in segmentation, tracking, or cell division detection, as well as to identify and manually annotate additional apoptotic events (Figure 1, Step 11). Features are automatically extracted from the segmented and tracked nuclei at each time point. Features include, among others, cell and nuclei morphometric parameters and GFP-MYC intensity distributions, as detailed in Supplementary Table S1. Tables are generated for each time-lapse experiment, where each row represents one cell at one particular time point. The number of cells analyzed varied from 14 to 145 per mESC colony culture.

Lineage tracing and context analysis. Cell identification by an ID allows relating dividing cells to their progeny, since each daughter cell ID carries information regarding its predecessors (Figure 2A). In addition, for every cell and time point, cell neighborhoods are identified as cells whose membranes are in direct contact with the cell of interest.

A specifically designed tool allows representation of cell lineages as trees for visualizing any of the features extracted from each cell and any time point within the lineage tree (Figure 2). The tool enables:

(i) Generating CSV files with selected cell families and calculation of parameters such as times to birth, division, and death.

(ii) Visualizing cell IDs and extracted features from the data table as lineage trees (Figure 2A); example features include mean nuclear GFP-MYC intensities (Figure 2B), GFP-MYC maximum intensity of neighbor cells (Figure 2C), and nuclear sizes (Figure 2D). Mean and maximum GFP-MYC intensities of neighbor cells are especially relevant for cell competition studies.

(iii) Filtering criteria for lineage tree visualization, such as temporal resolution, image quality, cell family identity, and number of families to be displayed.

Additionally, normalization of mean Myc values compensating for intensity loss during time is applied as described in the Supplementary Material. Once the tracking is completed for an entire image 
data set, aggregated measurements that use the whole-cell tracking information are computed for complete cell lifetimes, allowing the visualization of the trajectories of single cells together with representation of any parameter included in the feature table (Supplementary Table S1). Three outputs can be produced:

(i) Full trajectories and color-coded parameter values (Figure 2E), including position at each time point and cell division.

(ii) 2-D videos of the Z-projection of the tracked cell(s) with color-coded parameter values.

(iii) Combination of the previous two outputs in a single video where each frame shows the position of the cells and their feature value coded with colors, together with the trajectories followed from the beginning to the current time point (Figure 2F and Supplementary Video S1).

\section{Results and discussion}

We focused on studying Myc dynamics in the context of cell competition using live 4-D confocal images of mESCs expressing tdTomato and GFP-MYC. This required quantification of fluorescence intensity parameters across neighbor relationships and the integration of this neighboring analysis within the lineage trees based on prior automatic cell tracking from 4-D image data sets. One of the main challenges in acquiring live-cell 4-D images is optimizing spatial and temporal image resolution while minimizing photobleaching and phototoxicity, and allowing enough time to capture various cell processes (division, apoptosis, and cellcell interactions).

Live 4-D confocal imaging of Myc in mESCs required undersampling in the z-axis, which diminished resolving power. This clear limitation in axial resolution affected the image processing steps (Figure 3). The difficulty of obtaining accurate tracking results due to limitations in image resolution has been previously pointed out in several publications $(20,22)$. Besides the difficulties in automatic tracking of images that are greatly limited in axial and temporal resolution, the analysis of Myc dynamics also had additional drawbacks, such as the lack of cell division reporters and the use of GFP-MYC nuclear signal, which is maintained intentionally at very low physiological levels and presents a non-homogeneous intensity distribution throughout the cell population.

A

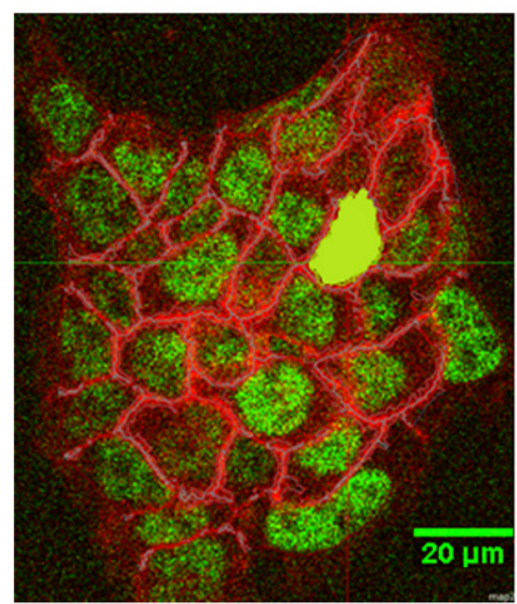

B

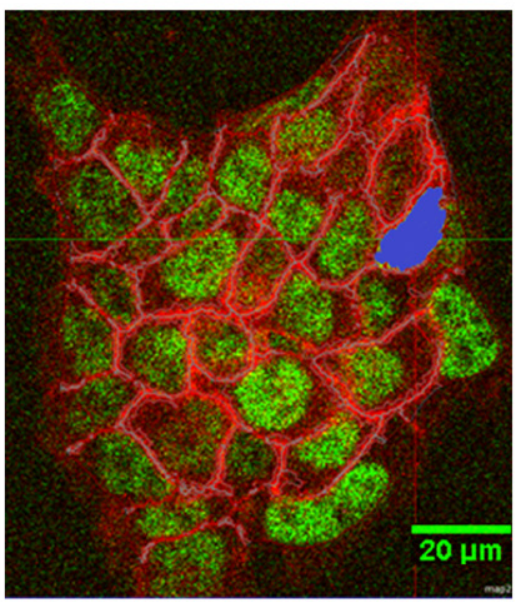

C
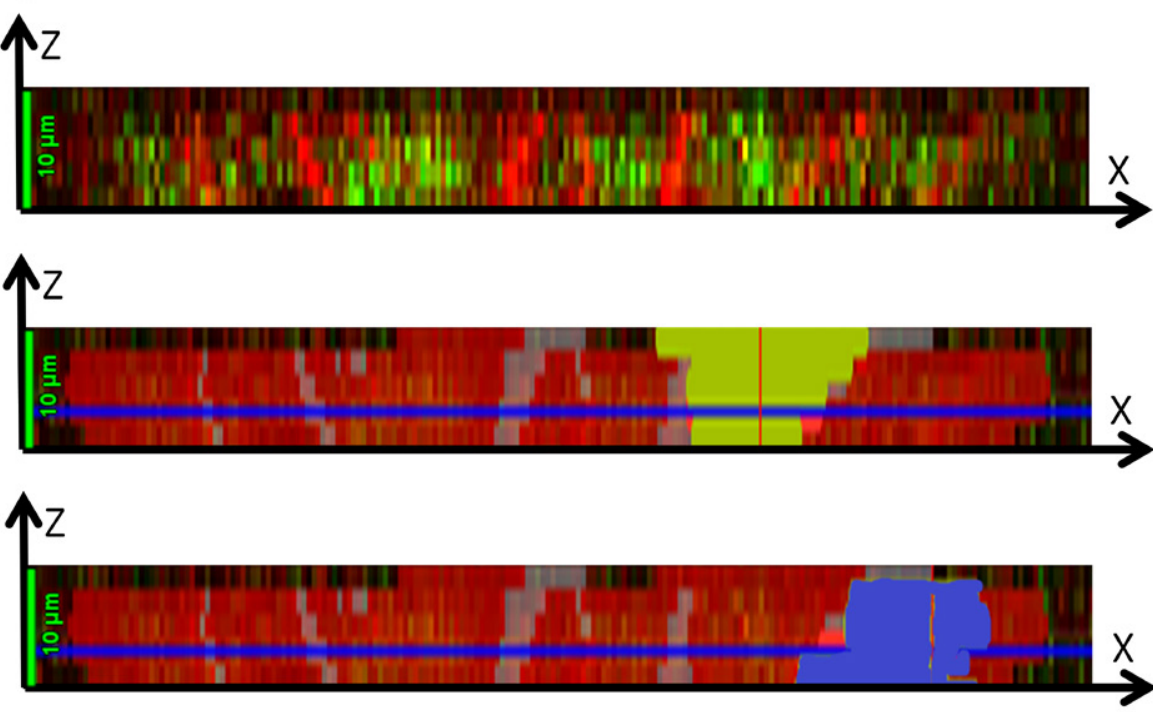

Figure 3. Z-stack confocal images from mouse embryonic stem cells (mESCs) 4-D image data sets, showing limitation in $x-, y$, and $z$-resolution. mESCs expressing GFP-MYC and tdTomato were imaged by confocal microscopy with $z$-sections spaced at $2 \mu \mathrm{m}$. Images show $x-y$ views of green (GFP-MYC) and red (tdTomato) signals, with the selected nuclei highlighted in yellow (A) and blue (B). (C) $x-z$ projection of 3-D Image $z$-stack and segmentation overlap for the nuclei highlighted in (A) and (B) (yellow and blue, respectively).

There are tracking packages that allow lineage reconstruction based on nuclear segmentation and thus require homogeneous nuclear signals, but these lack information on cellular contacts $(16,27,29)$. Analysis based on cellular membranes is absolutely necessary for the identification of cell-cell contacts, which is a prerequisite for the reconstruction of neighbor relationships necessary for the study of cell competition. Thus, our analysis required automatic cell segmentation and tracking based on both membrane and nuclear signals, where the nuclear signal is dim, non-homogeneous, and subject to intensity fluctuations. Several packages can perform lineage and neighbor analysis based on membrane signal (17-19,21,22,25); however, some of these packages either do not provide automatic tracking from those membrane images $(18,21,25)$, or require highlighting of dividing cells with additional specific fluorescent reporters (19). There are tracking methods that provide cell-based analysis, such as EDGE-D (17), which does not handle cell lineage tracing across cell division, or MARS-ALT (22), which requires scripting and does not offer integrated visualization of lineage trees, fluorescence markers, and neighborhood features. Therefore, our image analysis pipeline required several additional features compared with existing methods in order to allow automatic tracking of cells with nuclear intensity fluctuations, overcome limitations in axial and temporal resolution, not require homogenous nuclear staining or 


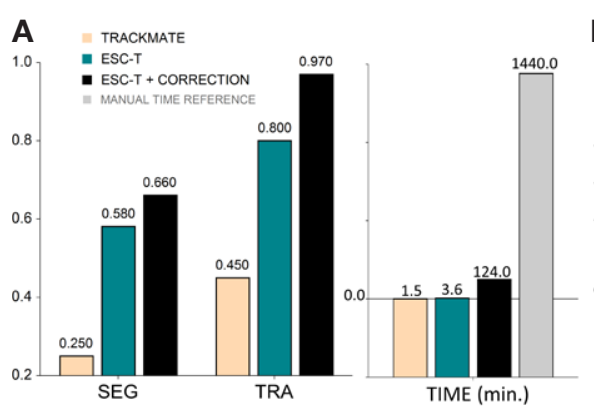

E

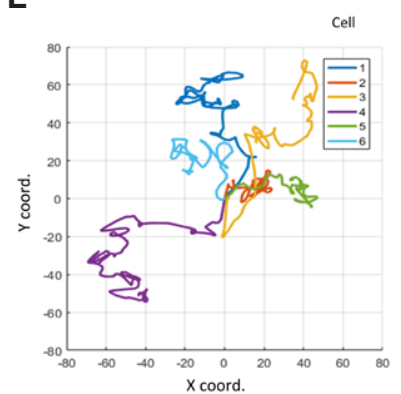

F ${ }_{5} \times 10^{-4}$
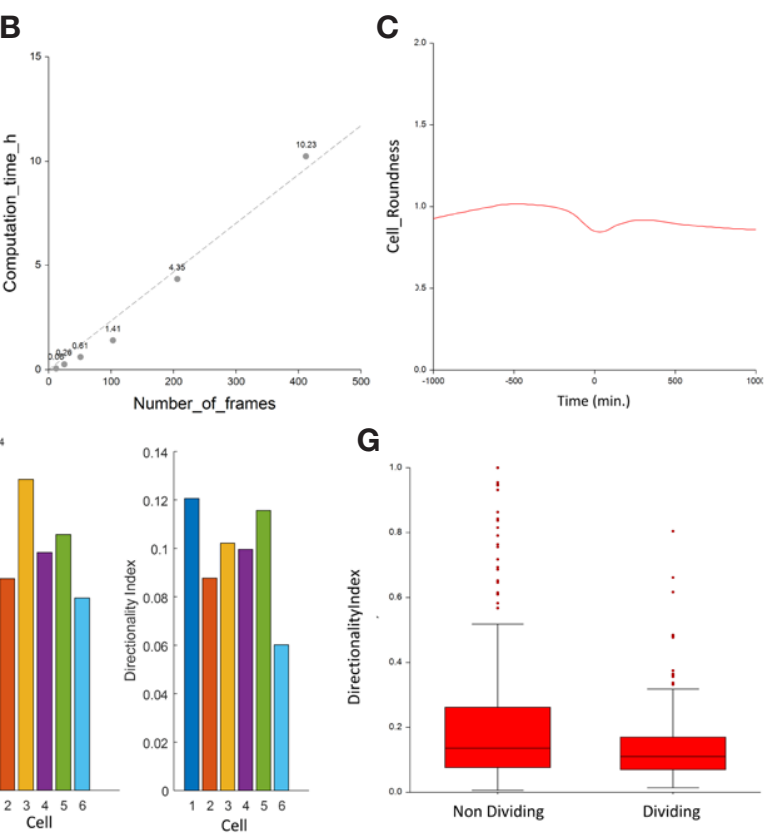

G

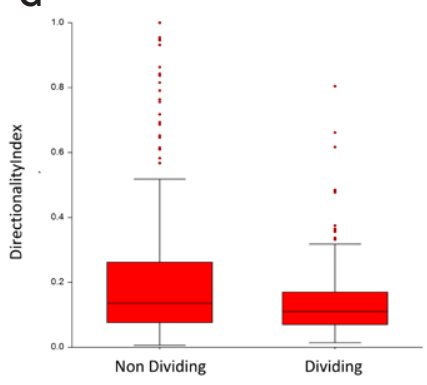

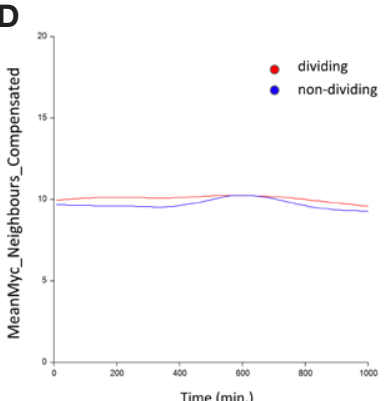

$\mathbf{H}$

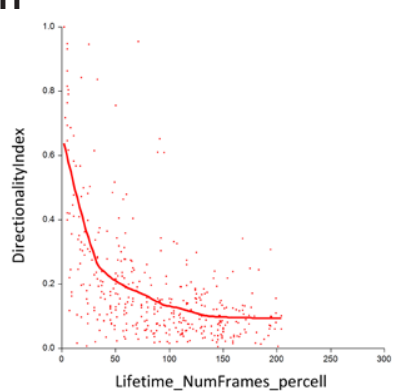

Figure 4. Embryonic stem cell tracking workflow (ESC-T) performance evaluation: cell shape, neighbor GFP-MYC level, and motion analysis after in silico synchronization to cell division time using ESC-T. (A) Performance of TRACKMATE or ESC-T fully automated processing and ESC-T including offline manual corrections of complete data set (ESC-T + corrections) was evaluated in terms of segmentation (SEG) and tracking (TRA) accuracy, using as reference the completely manual segmentation and tracking produced by an expert user. Execution times [TIME (min)] were measured for the fully automated processing with TRACKMATE, ESC-T or the offline corrected ESC-T (ESC-T + corrections), as well as for the manual procedure (manual time reference). (B) Computation times (in hours) for the segmentation and tracking of videos containing 12-412 frames, with an average of 14 cells in each frame. (C) Distribution of cell roundness (Supplementary Table S1) as a function of time from mitosis (min), obtained from live-recorded data, showing an increase of roundness during mitosis ( $P$ < 0.001). (D) Distribution of GFP-MYC levels of neighbors (MeanMyc_Neighbors_Compensated; Supplementary Table S1) after in silico classification of ESCs into dividing (red) and non-dividing (blue) cells according to live-recorded data as a function of distance to the beginning of each cell track. There were no significant differences between dividing and non-dividing cells $(P>0.001)$. Locally weighted scatterplot smoothing has been applied in (C) and (D). (E) Cell tracks of six randomly chosen cells with their origins aligned. (F) Analysis of the motility-related parameters of mean velocity and directionality index (effective distance/ walking_distance), derived from the individual cell tracks presented in (E) (color coding matches individual cell tracks). (G) Directionality index after in silico classification of cells as dividing or non-dividing. There were no significant differences between dividing and non-dividing cells $(P>0.001)$. $(H)$ Directionality index with respect to the (image-based) lifetime of every cell analyzed in a live-cell recording experiment. Locally weighted scatterplot smoothing has been applied.

mitosis highlighting, and provide integration of neighborhood and lineage relationships with tree visualization tools.

We deployed strategies based on the definition of extended forms of spatiotemporal connectivity rules in order to overcome limitations in axial resolution and in $z$-axis and time connectivity (Figure 1). Complex segmentation approaches based on 4-D mathematical morphology operators $(20,33)$ were discarded for the analysis of mESCS due to their sensitivity to spatiotemporal image resolution (20), leaving data association methods $(16,18)$ as the best option to handle the analysis. Moreover, the necessity of managing non-highlighted mitosis prevented the use of tools that do not directly handle actively dividing cells (17) or rely on highlighted mitotic spindles (19), leading to the requirement to manage cell segmentation based on feedback from the time dimension. This precluded the use of software based exclusively on segmentation in a time-independent manner, such as MARS (22), ACME(21), or RACE (18), and prompted us to implement a cell segmen- tation algorithm that was updated based on feedback from tracking and took into account biological knowledge such as the minimum temporal distance between two cell divisions or the nuclear shape before mitosis.

To establish a comparative study of the performance of ESC-T with respect to a tracking solution based on nuclear staining, we applied the ImageJ plugin TrackMate $(26,27)$. The gold standard was generated by manual segmentation and tracking of an image subset of 12 frames (1.4 h time lapse) of a colony of 14 cells. Segmentation and tracking accuracy was evaluated for the results obtained with TrackMate (defining the spherical detection as segmentation), ESC-T, and manually curated ESC-T (ESC-T + corrections). Segmentation (SEG) and tracking (TRA) metrics were computed to evaluate accuracy of the different methods using the benchmarking software provided by one of the cell tracking algorithm challenges (12) (detailed in the Supplementary Material). This revealed the superior performance of ESC-T compared with TrackMate (Figure 4A). The evaluation of execution times was carried out by measuring computation times with respect to number of frames, as described in the Supplementary Material. Execution times varied from $3.6 \mathrm{~min}$ to $10.23 \mathrm{~h}$ (Figure $4 \mathrm{~B})$, being the complexity of the algorithm $\mathrm{O}$ (n) (i.e., execution time of the segmentation and tracking algorithm is linear with respect to the number of video frames) (Pearson's correlation $\mathrm{R}>0.99 ; P<0.001$ ).

ESC-T was next used to study changes occurring during mitosis, when cells undergo a drastic cell rounding that is thought to facilitate organization within the mitotic cell and be necessary for the geometric requirements of division (30-32). These cell-shape changes were recapitulated when plotting the distribution of cell roundness as a function of time relative to mitotic completion (Figure 4C). ESC-T analysis showed the expected increase in cell roundness (defined in Supplementary Table S1) occurring during mitosis, with the values tending toward 0 (highest roundness) as cells approached cell division (0 distance to mitosis), in contrast to the stable distribution of roundness as the elapsed time before and after mitosis increased. 
the project, and did critical reading of the manuscript. M.C.M. contributed to the initial design of the ESC-T workflow, supervised the project, and wrote the manuscript.

\section{Acknowledgments}

We thank Antonio M. Santos, E. Arza and V. Labrador from the Advanced Imaging Unit for technical assistance in microscopy imaging. This work was supported by a grant from the Spanish Ministry of Economy and Competitiveness (MINECO) (ref: BIO201462200-EXP), and Innovative Training Networks (ITN), EU - H2020 programme (ref: 641639 BIOPOL) to M.C.M., grants BFU2015-71519-P and RD16/0011/0019 (ISCIII) from the Spanish Ministry of Economy and Competition (MINECO), and grant P2010/BMD-2315 from the Madrid Regional Government to M.T. C.D.D. was supported by a FPU grant from the Spanish Ministry of Education, Culture and Sports. The CNIC is supported by the Spanish Ministry of Economy and Competitiveness (MINECO) and the Pro CNIC Foundation, and is a Severo Ochoa Center of Excellence (MINECO award SEV-2015-0505). The ESC_Track software package and complete instructions for its use are freely available at www.cnic.es/cellomics/technology.

\section{Competing interests}

The authors declare no competing interests.

\section{References}

1. Huang, G., S. Ye, X. Zhou, D. Liu, and Q.L. Ying. 2015. Molecular basis of embryonic stem cell self-renewal: From signaling pathways to pluripotency network. Cell. Mol. Life Sci. 72:17411757.

2. Rossant, J. and P.P.L. Tam. 2017. New insights into early human development: lessons for stem cell derivation and differentiation. Cell Stem Cell 20:18-28.

3. Buckingham, M.E. and S.M. Meilhac. 2011. Tracing cells for tracking cell lineage and clonal behavior. Dev. Cell 21:394-409.

4. Kretzschmar, K. and F.M. Watt. 2012. Lineage tracing. Cell 148:33-45.

5. Blanpain, C. and B.D. Simons. 2013. Unravelling stem cell dynamics by lineage tracing. Nat. Rev. Mol. Cell Biol. 14:489-502.

6. Liu, Z. and P.J. Keller. 2016. Emerging Imaging and Genomic Tools for Developmental Systems Biology. Dev. Cell 36:597-610.

7. Pantazis, P. and W. Supatto. 2014. Advances in whole-embryo imaging: a quantitative transition is underway. Nat. Rev. Mol. Cell Biol. 15:327-339.

8. Specht, E.A., E. Braselmann, and A.E. Palmer. 2017. A critical and comparative review of fluorescent tools for live cell imaging. Annu Rev Physiol. 79:93-117.

9. Woodworth, M.B., K.M. Girskis, and C.A Walsh. 2017. Building a lineage from single cells: genetic techniques for cell lineage tracking. Nat. Rev. Gen_18:230-244

10. Meijering, E., A.E. Carpenter, H. Peng, F.A. Hamprecht, and J.-C. Olivo-Marin. 2016. Imagining the future of bioimage analysis. Nat. Biotechnol. 34:1250-1255.

11. Meijering, E., O. Dzyubachyk, I. Smal, and W.A. van Cappellen. 2009. Tracking in cell and developmental biology. Semin. Cell Dev. Biol. 20:894-902.

12. Maška, M., V. Ulman, D. Svoboda, P. Matula, P. Matula, C. Ederra, A. Urbiola, T. España, et al. 2014. A benchmark for comparison of cell tracking algorithms. Bioinformatics 30:1609-1617.

13. Bajcsy, P., M. Simon, S.J. Florczyk, C.G. Simon, D. Juba, and M.C. Brady. 2015. A method for the evaluation of thousands of automated 3-D stem cell segmentations. J. Microsc. 260:363-376.

14. Supatto, W., A. Mcmahon, S. Fraser, and A. Stathopoulos. 2009. Quantitative imaging of collective cell migration during Drosophila gastrulation: multiphoton microscopy and computational analysis. Nat. Protoc. 4:1397-1412.

15. Lemon, W.C., S.R. Pulver, B. Hockendorf, K. Mcdole, K. Branson, J. Freeman, and P.J. Keller. 2015. Whole-central nervous system functional imaging in larval Drosophila. Nat. Commun. 6:7924.

16. Amat, F., W. Lemon, D.P. Mossing, K. McDole, Y. Wan, K. Branson, E.W. Myers, and P.J.

\section{The SageHLS Hmw Library system \\ - Extract HMW DNA
from cell suspensions \\ - HLS-CATCH ${ }^{\mathrm{Tm}}$ - isolate genomic regions with CRISPR/Cas9}

for Long-Range Genomics

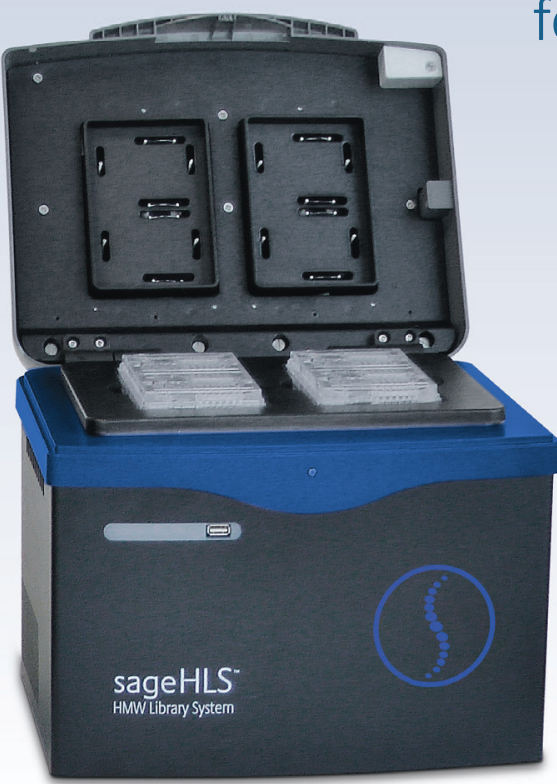

- Collect fragments up to $2 \mathrm{MB}$

- Less than 1 day workflow, minutes of hands-on time

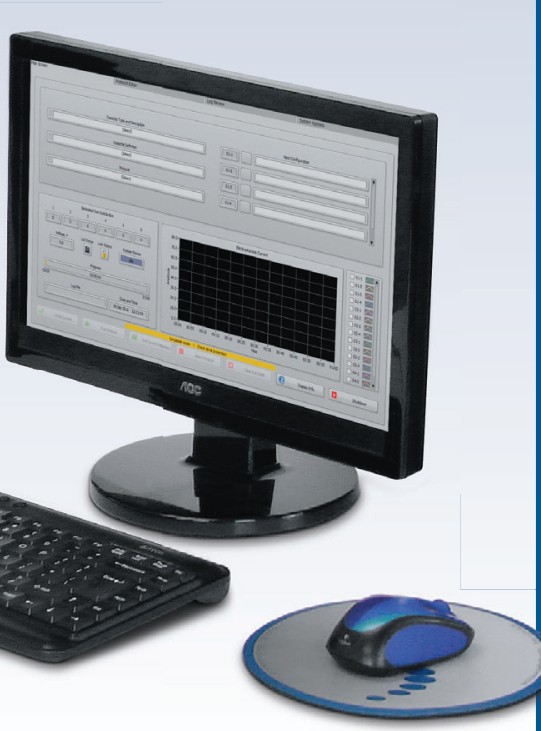


Keller. 2014. Fast, accurate reconstruction of cell lineages from large-scale fluorescence microscopy data. Nat. Methods 11:951-958.

17. Khan, Z., Y. Wang, E.F. Wieschaus, and M. Kaschube. 2014. Quantitative 4-D analyses of epithelial folding during Drosophila gastrulation. Development 141:2895-2900.

18. Stegmaier, J., F. Amat, W.C. Lemon, K. McDole, Y. Wan, G. Teodoro, R. Mikut, and P.J. Keller. 2016. Real-Time Three-Dimensional Cell Segmentation in Large-Scale Microscopy Data of Developing Embryos. Dev. Cell 36:225240.

19. Olivier, N., M.A. Luengo-Oroz, L. Duloquin, E. Faure, T. Savy, I. Veilleux, X. Solinas, D. Débarre, et al. 2010. Cell lineage reconstruction of early zebrafish embryos using labelfree nonlinear microscopy. Science 329:967-971.

20. Luengo-Oroz, M.A., D. Pastor-Escuredo, C. Castro-Gonzalez, E. Faure, T. Savy, B. Lombardot, J.L. Rubio-Guivernau, L. Duloquin, et al. 2012. Morphological processing: Applications to embryogenesis image analysis. IEEE Trans. Image Process. 21:3518-3530.

21. Mosaliganti, K.R., R.R. Noche, F. Xiong, I.A. Swinburne, and S.G. Megason. 2012. ACME: Automated cell morphology extractor for comprehensive reconstruction of cell membranes. PLOS Comput. Biol. 8:e1002780.

22. Fernandez, R., P. Das, V. Mirabet, E. Moscardi, J. Traas, J.-L. Verdeil, G. Malandain, and C. Godin. 2010. Imaging plant growth in 4-D: robust tissue reconstruction and lineaging at cell resolution. Nat. Methods 7:547-553.

23. de Chaumont, F., S. Dallongeville, N. Chenouard, N. Hervé, S. Pop, T. Provoost,
V. Meas-Yedid, P. Pankajakshan, et al. 2012. Icy: an open bioimage informatics platform for extended reproducible research. Nat. Methods 9:690-696.

24. Carpenter, A.E., T.R. Jones, M.R. Lamprecht, C. Clarke, I.H. Kang, O. Friman, D.A. Guertin, J.H. Chang, et al. 2006. CellProfiler: Image analysis software for identifying and quantifying cell phenotypes. Genome Biol. 7:R100.

25. Hilsenbeck, O., M. Schwarzfischer, S. Skylaki, B. Schauberger, P.S. Hoppe, D. Loeffler, K.D. Kokkaliaris, S. Hastreiter, et al. 2016. Software tools for single-cell tracking and quantification of cellular and molecular properties. Nat. Biotechnol. 34:703-706.

26. Schindelin, J., I. Arganda-Carreras, E. Frise, V. Kaynig, M. Longair, T. Pietzsch, S. Preibisch, C. Rueden, et al. 2012. Fiji: an open-source platform for biological-image analysis. Nat. Methods 9:676-682.

27. Tinevez, J.-Y., N. Perry, J. Schindelin, G.M. Hoopes, G.D. Reynolds, E. Laplantine, S.Y. Bednarek, S.L. Shorte, and K.W. Eliceiri. 2017. TrackMate: an open and extensible platform for single-particle tracking. Methods 115:80-90.

28. Jaqaman, K., D. Loerke, M. Mettlen, H. Kuwata, S. Grinstein, S.L. Schmid, and G. Danuser. 2008. Robust single-particle tracking in live-cell time-lapse sequences. Nat. Methods 5:695-702.

29. Murray, J.I., Z. Bao, T.J. Boyle, and R.H. Waterston. 2006. The lineaging of fluorescentlylabeled Caenorhabditis elegans embryos with StarryNite and AceTree. Nat. Protoc. 1:1468-1476.

30. Cramer, L.P. and T.J. Mitchison. 1997. Investigation of the mechanism of retraction of the cell margin and rearward flow of nodules during mitotic cell rounding. Mol. Biol. Cell 8:109-119.

31. Théry, M. and M. Bornens. 2006. Cell shape and cell division. Curr. Opin. Cell Biol. 18:648657.

32. Stewart, M.P., J. Helenius, Y. Toyoda, S.P. Ramanathan, D.J. Muller, and A.A. Hyman. 2011. Hydrostatic pressure and the actomyosin cortex drive mitotic cell rounding. Nature 469:226-230.

33. Bonneau, S., M. Dahan, and L.D. Cohen. 2005. Single quantum dot tracking based on perceptual Grouping using minimal paths in a spatiotemporal volume. IEEE Trans. Image Process. 14:1384-1395.

34. Scognamiglio, R., N. Cabezas-Wallscheid, M.C. Thier, S. Altamura, A. Reyes, Á.M. Prendergast, D. Baumgärtner, L.S. Carnevalli, et al. 2016. Myc Depletion Induces a Pluripotent Dormant State Mimicking Diapause. Cell 164:668-680.

35. Clavería, C., G. Giovinazzo, R. Sierra, and M. Torres. 2013. Myc-driven endogenous cell competition in the early mammalian embryo. Nature 500:39-44.

Received 23 December 2016; accepted 20 March 2017.

Address correspondence to María C. Montoya, Cellomics Unit, Cell \& Developmental Biology Area, Centro Nacional de Investigaciones Cardiovasculares CNIC. C/ Melchor Fernandez Almagro, 3. Madrid, E28029, Spain. E-mail: mmontoya@cnic.es

To purchase reprints of this article, contact: biotechniques@fosterprinting.com

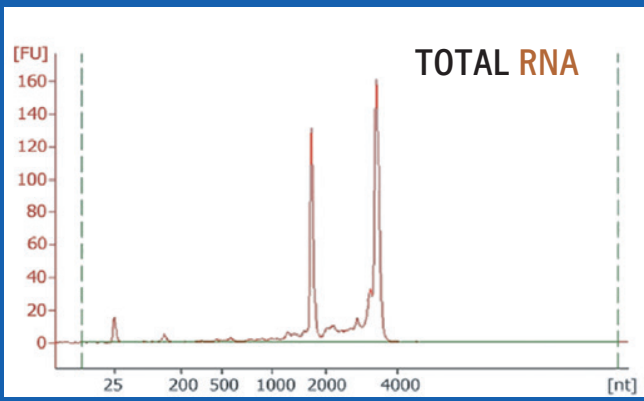

$\checkmark$ The single-step method without phase separation

No DNase treatment necessary

$\checkmark$ RNA ready for RT-PCR, microarrays and other applications

$\checkmark$ No need for refrigerated centrifuge

$\checkmark$ One reagent for solid and liquid samples

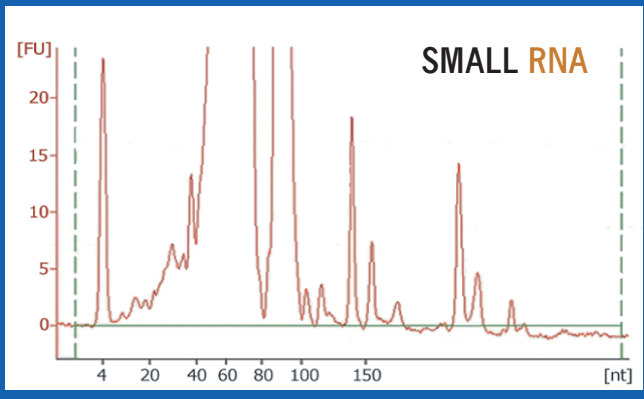

